\section{Event-based Sensor Data Scheduling: Trade-off Between Communication Rate and Estimation Quality}

\author{
Junfeng $\mathrm{Wu}^{\star}$, Qing-Shan $\mathrm{Jia}^{\dagger}$, Karl Henrik Johansson ${ }^{\sharp}$, Ling \\ $\mathrm{Shi}^{\star}$
}

\begin{abstract}
We consider sensor data scheduling for remote state estimation. Due to constrained communication energy and bandwidth, a sensor needs to decide whether it should send the measurement to a remote estimator for further processing. We propose an event-based sensor data scheduler for linear systems and derive the corresponding minimum squared error estimator. By selecting an appropriate eventtriggering threshold, we illustrate how to achieve a desired balance between the sensor-to-estimator communication rate and the estimation quality. Simulation examples are provided to demonstrate the theory.
\end{abstract}

Key words: Kalman filter; sensor scheduling; event-based scheduling; estimation performance.

\section{INTRODUCTION}

Networked control systems have received much attention in the last decade and are found in a wide spectrum of applications, e.g., in civil structure maintenance, environmental monitoring, battlefield surveillance. In many of these applications, sensor nodes are batterypowered. Replacing old batteries that are running out of energy are costly operations and may not even be possible. At the same time, the communication network may be shared by many nodes, and consequently the communication bandwidth might be scarce and uncertain. Thus it is practically important to minimize the sensorto-estimator communication rate. A too low rate may, however, lead to poor estimation quality. It is of significant interest to reduce the sensor-to-estimator communication rate while guarantee a certain level of desired estimation quality.

Related research on remote estimation under communication constraint and sensor scheduling in various forms have appeared in recent years. The problem of sensor scheduling can be traced back to the 1970s. Athans [1] first formulated a class of optimization problems dealing with selecting one measurement provided by one out of many sensors. Gupta et al. [2] proposed a stochastic sensor scheduling scheme among multiple sensors for one process and provided the optimal probability distribution over the sensors to be selected. In control of modern networked systems, actions are often desired to be taken only after certain events occur. These events may contain useful information about the system [3], and using an appropriate event-based scheduler, the performance of the estimator can be improved. Imer and Basar [4] considered optimal estimation with limited measurements where the stochastic process was a scalar linear system. They showed that the optimal observer policy has a solution in an event-triggered form. Cogill et al. [5] considered

$\star$ : Electronic and Computer Engineering, Hong Kong University of Science and Technology, Clear Water Bay, Kowloon, Hong Kong. Email: \{jfwu, eesling\}@ust.hk.

$\dagger$ : Center for Intelligent and Networked Systems, Department of Automation, TNLIST, Tsinghua University, Beijing, China. Email: jiaqs@ tsinghua.edu.cn.

\#: ACCESS Linnaeus Center, School of Electrical Engineering, Royal Institute of Technology, Stockholm, Sweden.Email: kallej@ee.kth.se.

The work by J. Wu and L. Shi is partially supported by a HK RGC CRF grant HKUST11/CRF/10 and a HK RGC GRF grant 618612. The work by Q.-S. Jia is supported in part by the National Science Foundation of China under grants (Nos. 60704008, 60736027, 61174072, and 90924001), the Specialized Research Fund for the Doctoral Program of Higher Education (No. 20070003110), the National 111 International Collaboration Project (No. B06002) and the TNLIST Cross Disciplinary Research Funding. The work by $\mathrm{K}$. H. Johansson is supported by the Knut and Alice Wallenberg Foundation and the Swedish Research Council.

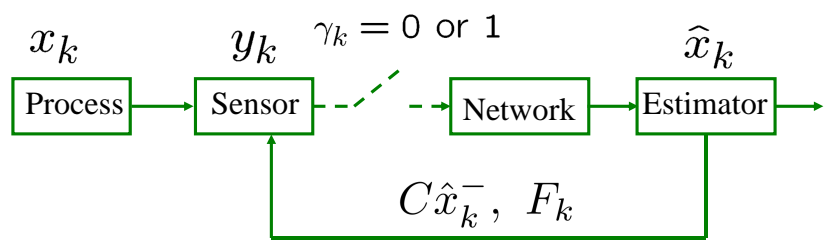

Fig. 1: Event-based Scheduling for Remote State Estimation

a sensor data scheduling problem and used a feedback policy to choose the transmission times which provides a trade-off between the communication rate and the estimation error. Ambrosino et al. [6] considered the channel capacity constraint. In recent work by Li et al. [7], an event-triggered approach was used to trigger the data transmission from a sensor to a remote observer in order to minimize the mean squared estimation error at the observer subject to a constraint on transmission frequency. Closely related works are also given by Riberio et al. [8] and Msechu et al. [9] where quantized Kalman filter were considered. The main distinctions between our work and [8], [9] include the different communication models (packed-based versus finite-bit channels) and different estimation procedures. While we design an event-based scheduler to optimize the tradeoff between the sensor-to-estimator communication rate and the remote estimation quality, the work of [8], [9] focused on designing encoder-decoder pairs to improve the estimation quality over a bit-limited channel.

This paper focuses on the design of sensor data scheduler and the corresponding networked state estimator illustrated by the architecture in Fig. 1. We propose an event-based sensor data scheduler and derive the corresponding minimum mean-squared error (MMSE) estimator. By adopting an approximation technique from nonlinear filtering, we derive a simple form of an accurate MMSE estimator, from which an illustrative relationship between the sensor-toestimator communication rate and the remote estimation quality can be obtained.

The remainder of this paper is organized as follows. In Section II, we provide the mathematical problem formulation. In Section III, we derive the exact MMSE estimator and an approximate MMSE estimator for an event-based sensor data scheduler. Via simulation examples in Section IV, we demonstrate how a desired trade-off between the sensor communication rate and the estimation quality can be achieved. It is also shown that the approximate MMSE estimator produces accurate results. In Section V, some concluding remarks are given.

Notation: $\mathbb{S}_{+}^{n}$ is the set of $n \times n$ positive semi-definite matrices. When $X \in \mathbb{S}_{+}^{n}$, we simply write $X \geq 0$; Similarly, $X \geq Y$ means $X-Y \geq 0 . f_{\mathrm{x}}(x)$ represents the probability density function (pdf) of the random variable (r.v.) $\mathrm{x}$, and $f_{\mathrm{x} \mid \mathrm{y}}(x \mid y)$ denotes the pdf of a r.v. $\mathrm{x}$ conditional on the variable y. $\mathcal{N}(\mu, \Sigma)$ denotes Gaussian distribution with mean $\mu$ and covariance matrix $\Sigma$. $\mathbb{E}[\cdot]$ denotes the mathematical expectation and $\operatorname{Pr}(\cdot)$ denotes the probability of a random event. $\operatorname{Tr}\{\cdot\}$ denotes the trace of a matrix and $\|\cdot\|_{\infty}$ denotes the Hölder infinity-norm of a vector.

\section{PRoblem SEtup}

\section{A. System Model}

Consider the following linear system:

$$
\begin{aligned}
\mathrm{x}_{k+1} & =A \mathrm{x}_{k}+w_{k}, \\
\mathrm{y}_{k} & =C \mathrm{x}_{k}+v_{k},
\end{aligned}
$$

where $\mathrm{x}_{k} \in \mathbb{R}^{n}$ is the state vector, $\mathrm{y}_{k} \in \mathbb{R}^{m}$ is the sensor measurement, $w_{k} \in \mathbb{R}^{n}$ and $v_{k} \in \mathbb{R}^{m}$ are mutually uncorrelated white Gaussian noises with covariances $Q \geq 0$ and $R>0$, respectively. 
The initial state $\mathrm{x}_{0}$ is zero-mean Gaussian with covariance matrix $\mathbb{E}\left[\mathrm{x}_{0} \mathrm{x}_{0}^{\prime}\right]=\Pi_{0} \geq 0$, and is uncorrelated with $w_{k}$ and $v_{k}$ for all $k \geq 0 .(A, C)$ and $(A, \sqrt{Q})$ are observable and controllable, respectively. After $y_{k}$ (the measured value of $\mathrm{y}_{k}$ ) is taken, the sensor decides whether it will send $y_{k}$ to a remote estimator for further processing. Let $\gamma_{k}=1$ or 0 be the decision variable whether $y_{k}$ shall be sent or not. Define $\mathrm{I}_{k} \triangleq\left\{\gamma_{0} y_{0}, \ldots, \gamma_{k} y_{k}\right\}$ with $\mathrm{I}_{-1} \triangleq \emptyset$,

$$
\hat{x}_{k}^{-} \triangleq \mathbb{E}\left[\mathrm{x}_{k} \mid \mathrm{I}_{k-1}\right], \mathrm{e}_{k}^{-} \triangleq \mathrm{x}_{k}-\hat{x}_{k}^{-}, P_{k}^{-} \triangleq \mathbb{E}\left[\mathrm{e}_{k}^{-} \mathrm{e}_{k}^{-'} \mid \mathrm{I}_{k-1}\right],
$$

and

$$
\hat{x}_{k} \triangleq \mathbb{E}\left[\mathrm{x}_{k} \mid \mathrm{I}_{k}\right], \quad \mathrm{e}_{k} \triangleq \mathrm{x}_{k}-\hat{x}_{k}, \quad P_{k} \triangleq \mathbb{E}\left[\mathrm{e}_{k} \mathrm{e}_{k}^{\prime} \mid \mathrm{I}_{k}\right]
$$

The estimates $\hat{x}_{k}^{-}$and $\hat{x}_{k}$ are called the a priori and a posteriori MMSE estimate, respectively. Further define the measurement innovation $\mathrm{Z}_{k}$ as

$$
\mathrm{z}_{k} \triangleq \mathrm{y}_{k}-\mathbb{E}\left[\mathrm{y}_{k} \mid \mathrm{I}_{k-1}\right]
$$

Define the functions $h, \tilde{g}_{\lambda}, g_{\lambda}$ and $g_{\lambda}^{k}: \mathbb{S}_{+}^{n} \rightarrow \mathbb{S}_{+}^{n}$ as follows:

$$
\begin{aligned}
\tilde{g}_{\lambda}(X) & \triangleq X-\lambda X C^{\prime}\left[C X C^{\prime}+R\right]^{-1} C X, \\
h(X) & \triangleq A X A^{\prime}+Q, \quad g_{\lambda}(X) \triangleq \tilde{g}_{\lambda} \circ h(X),
\end{aligned}
$$

where $\circ$ denotes the function composition. In the sequel, if $\lambda=1$, $g_{1}$ and $\tilde{g}_{1}$ will be written as $g$ and $\tilde{g}$ for brevity. We can write the update equation for $P_{k}$ in a compact form as

$$
P_{k}= \begin{cases}g\left(P_{k-1}\right), & \text { if } \gamma_{k}=1, \\ h\left(P_{k-1}\right), & \text { if } \gamma_{k}=0 .\end{cases}
$$

Notice that $h\left(P_{k-1}\right) \geq g\left(P_{k-1}\right)$ as $\tilde{g}(X) \leq X$ for any $X \geq 0$. This has an intuitive explanation: the measurement $\mathrm{y}_{k}$ (or alternatively, the innovation $\mathrm{z}_{k}$ ) always reduces the estimation error covariance.

\section{B. Event-based Sensor Scheduler}

We consider in this paper applications where feedback is available from the estimator to the sensor, see Fig. 1. ${ }^{1}$

Consider the following two cases for the Kalman filter when $z_{k}=$ 0 :

1) $\gamma_{k}=1: \hat{x}_{k}=A \hat{x}_{k-1}$ and $P_{k}=g\left(P_{k-1}\right)$,

2) $\gamma_{k}=0: \hat{x}_{k}=A \hat{x}_{k-1}$ and $P_{k}=h\left(P_{k-1}\right)$.

The estimate $\hat{x}_{k}$ for the two cases are the same, but the error covariances are different. Therefore if the sensor finds that $z_{k}$ is zero and does not send $y_{k}$ to the estimator, and at the same time, the estimator is aware of this information, then even without receiving $y_{k}$, the estimator knows that $\hat{x}_{k}=A \hat{x}_{k-1}$ has error covariance $g\left(P_{k-1}\right)$, which is smaller than $h\left(P_{k-1}\right)$.

Since $C P_{k}^{-} C^{\prime}+R>0$, there exists a unitary matrix $U_{k} \in$ $\mathbb{R}^{m \times m}$ such that

$$
U_{k}^{\prime}\left(C P_{k}^{-} C^{\prime}+R\right) U_{k}=\Lambda_{k},
$$

where $\Lambda_{k}=\operatorname{diag}\left(\lambda_{k}^{1}, \ldots, \lambda_{k}^{m}\right) \in \mathbb{R}^{m \times m}$ and $\lambda_{k}^{1}, \ldots, \lambda_{k}^{m} \in \mathbb{R}$ are the eigenvalues of $C P_{k}^{-} C^{\prime}+R$. Define $F_{k} \in \mathbb{R}^{m \times m}$ as

$$
F_{k} \triangleq U_{k} \Lambda_{k}^{-\frac{1}{2}}
$$

\footnotetext{
${ }^{1}$ Examples of such applications can be found in remote estimation based on the IEEE 802.15.4/ZigBee protocol: sensor devices can be scheduled to communicate to the so-called Personal Area Network coordinator which also serves as a remote estimator. The coordinator broadcasts information to all devices at the beginning of each periodic superframe and can then incorporate the required feedback information.
}

Evidently, $F_{k}^{\prime} F_{k}=\left(C P_{k}^{-} C^{\prime}+R\right)^{-1}$. The matrix $F_{k}$ is computed by the remote estimator and is sent back to the sensor along with $C \hat{x}_{k}^{-}$at each time, see Fig. 1. Define $\epsilon_{k}$ as

$$
\epsilon_{k} \triangleq F_{k}^{\prime} \mathrm{z}_{\mathrm{k}}
$$

This transformation is called the Mahalanobis transformation. The coordinates of $\mathrm{z}_{\mathrm{k}}$ are decorrelated, so $\epsilon_{k}$ has $m$-variable standard Gaussian distribution, which contains a set of independent principal components of $\mathrm{Z}_{\mathrm{k}}$.

We consider the following event-based sensor data scheduler:

$$
\gamma_{k}= \begin{cases}0, & \text { if }\left\|\epsilon_{k}\right\|_{\infty} \leq \delta \\ 1, & \text { otherwise }\end{cases}
$$

where $\delta \geq 0$ is a fixed threshold. Under this scheduler, if $\gamma_{k}=0$, the estimator can infer that $\left\|\epsilon_{k}\right\|_{\infty} \leq \delta$. It is this additional information that helps reduce the estimation error at the remote estimator. With a slight abuse of notation, we redefine the information $\mathrm{I}_{k}$ received by the remote estimator till $k$ as

$$
\mathrm{I}_{k} \triangleq\left\{\gamma_{0} y_{0}, \ldots, \gamma_{k} y_{k}\right\} \cup\left\{\gamma_{0}, \ldots, \gamma_{k}\right\}
$$

Define the average sensor communication rate as

$$
\gamma \triangleq \limsup _{T \rightarrow+\infty} \frac{1}{T+1} \sum_{k=0}^{T} \mathbb{E}\left[\gamma_{k}\right]
$$

Notice that both the average rate $\gamma$ and the estimation error covariance matrix $P_{k}$ depend on the threshold $\delta$. For example, if $\delta=0$, then $\operatorname{Pr}\left(\left\|\epsilon_{k}\right\|_{\infty} \leq 0\right)=0$ and the sensor sends $y_{k}$ at each $k$ (almost surely). Consequently, $\gamma=1$ and $P_{k}=g\left(P_{k-1}\right)$. On the other hand, if $\delta=+\infty$, then the sensor keeps $y_{k}$ for all $k$, thus making $\gamma=0$. As the event $\left\|\epsilon_{k}\right\|_{\infty} \leq+\infty$ provides no extra information on the innovation, the estimator is in this case equivalent to an openloop predictor. Therefore, $P_{k}=h\left(P_{k-1}\right)$. In the latter case, when $A$ is unstable, $P_{k}$ diverges as $k \rightarrow+\infty$. Apparently there is a trade-off between the communication rate and the estimation quality.

We now state the main problems considered in this paper.

1) Under the event-based sensor scheduler (8), what is the MMSE estimator?

2) How to choose the threshold $\delta$ in (8) to achieve a desirable trade-off between the communication rate and the estimation quality?

We will provide answers to these two problems in the remainder of the paper.

\section{EVEnT-BAsEd StATE Estimation}

In this section, we derive the MMSE estimator under the eventbased sensor data scheduler (8), first the exact estimator and then an accurate approximation.

\section{A. The Exact MMSE Estimator}

The MMSE estimate is uniquely specified as the conditional mean given all available information [10]. In this subsection, we provide an exact MMSE estimator corresponding to the event-based scheduler (8) using the following two-step updating procedure.

1. Time Update: The a priori estimate $\hat{x}_{k}^{-}$, which is the conditional mean of $\mathrm{x}_{k}$ given the information set $\mathrm{I}_{k-1}$, is derived as

$$
\hat{x}_{k}^{-}=\mathbb{E}\left[\mathrm{x}_{k} \mid \mathrm{I}_{k-1}\right]=\int_{\mathbb{R}^{m}} x f_{\mathrm{x}_{k}}\left(x \mid \mathrm{I}_{k-1}\right) \mathrm{d} x,
$$

and the corresponding estimation error covariance $P_{k}^{-}$is given by

$$
P_{k}^{-}=\int_{\mathbb{R}^{m}}\left(x-\hat{x}_{k}^{-}\right)\left(x-\hat{x}_{k}^{-}\right)^{\prime} f_{\mathrm{x}_{k}}\left(x \mid \mathrm{I}_{k-1}\right) \mathrm{d} x .
$$


2. Measurement Update: The a posteriori estimate $\hat{x}_{k}$, which is the conditional mean of $\mathrm{x}_{k}$ given $\mathrm{I}_{k}$, is derived as follows. Depending on whether $\gamma_{k}=0$ or 1 , we have the following two cases:

1) $\gamma_{k}=0$. The sensor does not send $y_{k}$ to the remote estimator, but the estimator is aware of that $\left\|\epsilon_{k}\right\|_{\infty} \leq \delta$. Consequently, $\hat{x}_{k}$ is given by

$$
\hat{x}_{k}=\mathbb{E}\left[\mathrm{x}_{k} \mid \hat{\mathrm{I}}_{k}\right]=\int_{\mathbb{R}^{m}} x f_{\mathrm{x}_{k}}\left(x \mid \hat{\mathrm{I}}_{k}\right) \mathrm{d} x,
$$

where we denote $\hat{\mathrm{I}}_{k}=\mathrm{I}_{k-1} \cup\left\{\gamma_{k}=0\right\}$. Define the set $\Omega \subset \mathbb{R}^{m}$ as

$$
\Omega \triangleq\left\{\epsilon_{k} \in \mathbb{R}^{m}:\left\|\epsilon_{k}\right\|_{\infty} \leq \delta\right\},
$$

then one can compute $f_{\mathrm{x}_{k}}\left(x \mid \hat{\mathrm{I}}_{k}\right)$ using Bayes' rule as

$$
f_{\mathrm{x}_{k}}\left(x \mid \hat{\mathrm{I}}_{k}\right)=\frac{f_{\mathrm{x}_{k}}\left(x \mid \mathrm{I}_{k-1}\right) \int_{\Omega} f_{\epsilon_{k}}\left(\epsilon \mid \mathrm{I}_{k-1}, \mathrm{x}_{k}\right) \mathrm{d} \epsilon}{\int_{\Omega} f_{\epsilon_{k}}\left(\epsilon \mid \mathrm{I}_{k-1}\right) \mathrm{d} \epsilon},
$$

where $f_{\epsilon_{k}}\left(\epsilon \mid \mathrm{I}_{k-1}, \mathrm{x}_{k}\right)=\mathcal{N}\left(F_{k}^{\prime} \mathrm{x}_{k}-F_{k}^{\prime} C \hat{x}_{k}^{-}, F_{k}^{\prime} R F_{k}\right)$ and

$$
\mathrm{z}_{k}=C \mathrm{x}_{k}+v_{k}-C \hat{x}_{k}^{-}=C \mathrm{e}_{k}^{-}+v_{k} .
$$

The a posteriori error covariance $P_{k}$ is given by

$$
P_{k}=\int_{\mathbb{R}^{m}}\left(x-\hat{x}_{k}\right)\left(x-\hat{x}_{k}\right)^{\prime} f_{\mathrm{x}_{k}}\left(x \mid \hat{\mathrm{I}}_{k}\right) \mathrm{d} x .
$$

2) $\gamma_{k}=1$. The sensor sends $y_{k}$ to the remote estimator. Denote the measured value of the innovation $\mathrm{z}_{k}$ as $z$. Then $\mathrm{I}_{k}$ becomes $\mathrm{I}_{k}=\mathrm{I}_{k-1} \cup\left\{\mathrm{z}_{k}=z\right\}$. The remote estimator updates $\hat{x}_{k}$ as in (11), but the conditional pdf $f_{\mathrm{x}_{k}}\left(x \mid \mathrm{I}_{k}\right)$ is now calculated using Bayes' rule as

$$
f_{\mathrm{x}_{k}}\left(x \mid \mathrm{I}_{k}\right)=\frac{f_{\mathrm{x}_{k}}\left(x \mid \mathrm{I}_{k-1}\right) f_{\mathrm{z}_{k}}\left(z \mid \mathrm{I}_{k-1}, \mathrm{x}_{k}\right)}{f_{\mathrm{z}_{k}}\left(z \mid \mathrm{I}_{k-1}\right)},
$$

where, from (14), one easily sees that

$$
f_{\mathrm{z}_{k}}\left(z \mid \mathrm{I}_{k-1}, \mathrm{x}_{k}\right)=\mathcal{N}\left(C \mathrm{x}_{k}-C \hat{x}_{k}^{-}, R\right) .
$$

The a posteriori estimation error covariance $P_{k}$ is given by

$$
P_{k}=\int_{\mathbb{R}^{m}}\left(x-\hat{x}_{k}\right)\left(x-\hat{x}_{k}\right)^{\prime} f_{\mathrm{x}_{k}}\left(x \mid \mathrm{I}_{k-1}, \mathrm{z}_{k}=z\right) \mathrm{d} x .
$$

Remark 3.1. Although the above two steps produce the MMSE estimate $\hat{x}_{k}$ corresponding to the event-based scheduler (8), each updating step requires numerical integration. The amount of computation involved make this estimator intractable in general, which motivates us to consider an approximate MMSE estimator. As we will demonstrate, by using a standard technique in nonlinear filtering, we can derive an approximate MMSE estimator in a simple recursive form.

\section{B. Approximate MMSE Estimator}

A commonly used approximation technique in nonlinear filtering is to assume that the conditional distribution of $\mathrm{x}_{k}$ given $\mathrm{I}_{k-1}$ is Gaussian, i.e.,

$$
f_{\mathrm{x}_{k}}\left(x \mid \mathrm{I}_{k-1}\right)=\mathcal{N}\left(\hat{x}_{k}^{-}, P_{k}^{-}\right) .
$$

This assumption reduces the estimation problem from the tracking of a general pdf, which is usually computationally intractable, to the tracking of its mean and covariance matrix. The approximation is widely used in the literature, e.g., [8], [9], [11]. Unless specifically mentioned, our analysis in the rest of this paper is based on this assumption. The approximation leads to a very simple form of the estimator, as shown by the following result.

Theorem 3.2. Consider the remote state estimation in Fig. 1 with the event-based sensor scheduler (8). Under the assumption (16), the MMSE estimator is given recursively as follows:

1) Time update:

$$
\left\{\begin{array}{l}
\hat{x}_{k}^{-}=A \hat{x}_{k-1} \\
P_{k}^{-}=h\left(P_{k-1}\right) .
\end{array}\right.
$$

2) Measurement update:

$$
\left\{\begin{array}{l}
\hat{x}_{k}=\hat{x}_{k}^{-}+\gamma_{k} L_{k} z_{k} \\
P_{k}=\gamma_{k} \tilde{g}\left(P_{k}^{-}\right)+\left(1-\gamma_{k}\right) \tilde{g}_{\beta(\delta)}\left(P_{k}^{-}\right)
\end{array}\right.
$$

where

$$
\beta(\delta)=\frac{2}{\sqrt{2 \pi}} \delta e^{-\frac{\delta^{2}}{2}}[1-2 Q(\delta)]^{-1},
$$

and $Q(\cdot)$ is the standard $Q$-function defined by

$$
Q(\delta) \triangleq \int_{\delta}^{+\infty} \frac{1}{\sqrt{2 \pi}} e^{-\frac{x^{2}}{2}} \mathrm{~d} x .
$$

Before we present the proof, we state a few preliminary results.

From (14) and (16), $\mathrm{z}_{k}$ is zero-mean Gaussian conditioned on $\mathrm{I}_{k-1}$. Furthermore, $\mathrm{z}_{k}$ is jointly Gaussian with $\mathrm{x}_{k}$ conditioned on $\mathrm{I}_{k-1}$. From (14),

$$
\mathbb{E}\left[\mathrm{z}_{k} \mathrm{z}_{k}^{\prime} \mid \mathrm{I}_{k-1}\right]=C \mathbb{E}\left[\mathrm{e}_{k}^{-} \mathrm{e}_{k}^{-'} \mid \mathrm{I}_{k-1}\right] C^{\prime}+R=C P_{k}^{-} C^{\prime}+R,
$$

and

$$
\mathbb{E}\left[\mathrm{e}_{k}^{-} \mathrm{z}_{k}^{\prime} \mid \mathrm{I}_{k-1}\right]=\mathbb{E}\left[\mathrm{e}_{k}^{-} \mathrm{e}_{k}^{-{ }^{\prime}} \mid \mathrm{I}_{k-1}\right] C^{\prime}=P_{k}^{-} C^{\prime} .
$$

Now let us take a look at $\epsilon_{k}$ defined in (7). From (20),

$$
\mathbb{E}\left[\epsilon_{k} \epsilon_{k}^{\prime} \mid \mathrm{I}_{k-1}\right]=F_{k}^{\prime} \mathbb{E}\left[\mathrm{Z}_{\mathrm{k}} \mathrm{Z}_{\mathrm{k}}{ }^{\prime} \mid \mathrm{I}_{k-1}\right] F_{k}=I_{m}
$$

Thus, given $\mathrm{I}_{k-1}, \epsilon_{k}$ is a zero-mean Gaussian multivariate random variable with unit variance. Denote $\epsilon_{k}^{i}$ as the $i$ th element of $\epsilon_{k}$. Then $\epsilon_{k}^{i}$ and $\epsilon_{k}^{j}$ are mutually independent if $i \neq j$. Notice that $\gamma_{k}=$ 0 implies that the event $\left\|\epsilon_{k}\right\|_{\infty} \leq \delta$ happens. We then have the following result.

Lemma 3.3. $F_{k}^{\prime} \mathbb{E}\left[\mathrm{z}_{k} \mathrm{z}_{k}^{\prime} \mid \hat{\mathrm{I}}_{k}\right] F_{k}=\mathbb{E}\left[\epsilon_{k} \epsilon_{k}^{\prime} \mid \hat{\mathrm{I}}_{k}\right]=[1-\beta(\delta)] I_{m}$.

Proof: Straightforward calculation yields the first equality. Given $\mathrm{I}_{k-1}$, due to the independence of $\epsilon_{k}^{i}$ and $\epsilon_{k}^{j}$ for $i \neq j$ along with Lemma A.1 in the Appendix, we have

$$
\begin{aligned}
\mathbb{E}\left[\left(\epsilon_{k}^{i}\right)^{2} \mid \hat{\mathrm{I}}_{k}\right] & =\mathbb{E}\left[\left(\epsilon_{k}^{i}\right)^{2} \mid \mathrm{I}_{k-1},\left\|\epsilon_{k}\right\|_{\infty} \leq \delta\right] \\
& =\mathbb{E}\left[\left(\epsilon_{k}^{i}\right)^{2}\left|\mathrm{I}_{k-1},\right| \epsilon_{k}^{i} \mid \leq \delta\right]=1-\beta(\delta)
\end{aligned}
$$

and

$$
\mathbb{E}\left[\epsilon_{k}^{i} \epsilon_{k}^{j} \mid \hat{\mathrm{I}}_{k}\right]=\mathbb{E}\left[\epsilon_{k}^{i} \epsilon_{k}^{j}\left|\mathrm{I}_{k-1},\right| \epsilon_{k}^{i}|\leq \delta,| \epsilon_{k}^{j} \mid \leq \delta\right]=0 .
$$

Thus,

$$
\mathbb{E}\left[\epsilon_{k} \epsilon_{k}{ }^{\prime} \mid \hat{\mathrm{I}}_{k}\right]=[1-\beta(\delta)] I_{m}
$$

The following lemma is used in deriving the main result.

Lemma 3.4. The following equalities hold.

$$
\begin{aligned}
& \mathbb{E}\left[\mathrm{e}_{k}^{-} \mathrm{z}_{k}^{\prime} \mid \hat{\mathrm{I}}_{k}\right]=L_{k} \mathbb{E}\left[\mathrm{z}_{k} \mathrm{z}_{k}^{\prime} \mid \hat{\mathrm{I}}_{k}\right], \\
& \mathbb{E}\left[\left(\mathrm{e}_{k}^{-}-L_{k} \mathrm{z}_{k}\right) \mathrm{z}_{k}^{\prime} \mid \hat{\mathrm{I}}_{k}\right]=0, \\
& \mathbb{E}\left[\left(\mathrm{e}_{k}^{-}-L_{k} \mathrm{z}_{k}\right)\left(\mathrm{e}_{k}^{-}-L_{k} \mathrm{z}_{k}\right)^{\prime} \mid \mathrm{I}_{k-1}, \mathrm{z}_{k}=z\right]=\tilde{g}\left(P_{k}^{-}\right), \\
& \mathbb{E}\left[\left(\mathrm{e}_{k}^{-}-L_{k} \mathrm{z}_{k}\right)\left(\mathrm{e}_{k}^{-}-L_{k} \mathrm{z}_{k}\right)^{\prime} \mid \hat{\mathrm{I}}_{k}\right]=\tilde{g}\left(P_{k}^{-}\right),
\end{aligned}
$$


where $L_{k}=P_{k}^{-} C^{\prime}\left[C P_{k}^{-} C^{\prime}+R\right]^{-1}$.

Proof: We first prove (22). From Lemma A.2, (20) and (21),

$$
\mathbb{E}\left[\mathrm{x}_{k} \mid \mathrm{I}_{k-1}, \mathrm{z}_{k}=z\right]=\hat{x}_{k}^{-}+P_{k}^{-} C^{\prime}\left[C P_{k}^{-} C^{\prime}+R\right]^{-1} z .
$$

Since given $\mathrm{I}_{k-1}, \epsilon_{k}$ is Gaussian with zero mean and unit covariance, we can define $p_{\delta} \triangleq \operatorname{Pr}\left(\left\|\epsilon_{k}\right\|_{\infty} \leq \delta \mid \mathrm{I}_{k-1}\right)$. Using the conditional pdf

$$
f_{\epsilon_{k}}\left(\epsilon \mid \hat{\mathrm{I}}_{k}\right)= \begin{cases}\frac{f_{\epsilon_{k}}\left(\epsilon \mid \mathrm{I}_{k-1}\right)}{p_{\delta}}, & \text { if }\left\|\epsilon_{k}\right\| \leq \delta ; \\ 0, & \text { otherwise; }\end{cases}
$$

we obtain

$$
\begin{aligned}
& \mathbb{E}\left[\mathrm{e}_{k}^{-} \mathrm{z}_{k}^{\prime} \mid \hat{\mathrm{I}}_{k}\right] \\
& =\frac{1}{p_{\delta}} \int_{\Omega} \mathbb{E}\left[\mathrm{e}_{k}^{-} \mid \mathrm{I}_{k-1}, \mathrm{z}_{k}=F_{k}^{\prime-1} \epsilon\right] \epsilon^{\prime} F_{k}^{-1} f_{\epsilon_{k}}\left(\epsilon \mid \mathrm{I}_{k-1}\right) \mathrm{d} \epsilon \\
& =\frac{1}{p_{\delta}} \int_{\Omega} \mathbb{E}\left[\mathrm{x}_{k}-\hat{x}_{k}^{-} \mid \mathrm{I}_{k-1}, \mathrm{z}_{k}=F_{k}^{\prime-1} \epsilon\right] \epsilon^{\prime} F_{k}^{-1} f_{\epsilon_{k}}\left(\epsilon \mid \mathrm{I}_{k-1}\right) \mathrm{d} \epsilon \\
& =\frac{1}{p_{\delta}} \int_{\Omega}\left(\mathbb{E}\left[\mathrm{x}_{k} \mid \mathrm{I}_{k-1}, \mathrm{z}_{k}=F_{k}^{\prime-1} \epsilon\right]-\hat{x}_{k}^{-}\right) \epsilon^{\prime} F_{k}^{-1} f_{\epsilon_{k}}\left(\epsilon \mid \mathrm{I}_{k-1}\right) \mathrm{d} \epsilon \\
& =P_{k}^{-} C^{\prime}\left(C P_{k}^{-} C^{\prime}+R\right)^{-1} F_{k}^{\prime-1} \frac{1}{p_{\delta}} \int_{\Omega} \epsilon \epsilon_{\epsilon_{k}}\left(\epsilon \mid \mathrm{I}_{k-1}\right) \mathrm{d} \epsilon F_{k}^{-1} \\
& =L_{k} F_{k}^{\prime-1} \mathbb{E}\left[\epsilon_{k} \epsilon_{k}^{\prime} \mid \hat{\mathrm{I}}_{k}\right] F_{k}^{-1} \\
& =L_{k} \mathbb{E}\left[\mathrm{z}_{k} \mathrm{z}_{k}^{\prime} \mid \hat{\mathrm{I}}_{k}\right],
\end{aligned}
$$

where the last equality is from Lemma 3.3. From (22) we have

$$
\mathbb{E}\left[\left(\mathrm{e}_{k}^{-}-L_{k} \mathrm{z}_{k}\right) \mathrm{z}_{k}^{\prime} \mid \hat{\mathrm{I}}_{k}\right]=\mathbb{E}\left[\mathrm{e}_{k}^{-} \mathrm{z}_{k}^{\prime} \mid \hat{\mathrm{I}}_{k}\right]-L_{k} \mathbb{E}\left[\mathrm{z}_{k} \mathrm{z}_{k}^{\prime} \mid \hat{\mathrm{I}}_{k}\right]=0,
$$

which shows (23). To prove (24), using Lemma A.2, we have

$$
\begin{aligned}
& \mathbb{E}\left[\left(\mathrm{x}_{k}-\mathbb{E}\left[\mathrm{x}_{k} \mid \mathrm{I}_{k-1}, \mathrm{z}_{k}=z\right]\right)\left(\mathrm{x}_{k}-\mathbb{E}\left[\mathrm{x}_{k} \mid \mathrm{I}_{k-1}, \mathrm{z}_{k}=z\right]\right)^{\prime} \mid \mathrm{I}_{k-1}, \mathrm{z}_{k}=z\right] \\
& =P_{k}^{-}-P_{k}^{-} C^{\prime}\left(C P_{k}^{-} C^{\prime}+R\right)^{-1} C P_{k}^{-}=\tilde{g}\left(P_{k}^{-}\right) .
\end{aligned}
$$

Notice that (26) leads to

$$
\mathrm{x}_{k}-\mathbb{E}\left[\mathrm{x}_{k} \mid \mathrm{I}_{k-1}, \mathrm{z}_{k}=z\right]=\mathrm{x}_{k}-\hat{x}_{k}^{-}-L_{k} z=\mathrm{e}_{k}^{-}-L_{k} z,
$$

which together with (28) shows (24). Now from (24), one obtains

$$
\begin{aligned}
& \mathbb{E}\left[\left(\mathrm{e}_{k}^{-}-L_{k} \mathrm{z}_{k}\right)\left(\mathrm{e}_{k}^{-}-L_{k} \mathrm{z}_{k}\right)^{\prime} \mid \hat{\mathrm{I}}_{k}\right] \\
= & \int_{\Omega} \mathbb{E}\left[\left(\mathrm{e}_{k}^{-}-L_{k} \mathrm{z}_{k}\right)\left(\mathrm{e}_{k}^{-}-L_{k} \mathrm{z}_{k}\right)^{\prime} \mathrm{I}_{k-1}, \mathrm{z}_{k}=F_{k}^{\prime-1} \epsilon\right] \frac{f_{\epsilon_{k}}\left(\epsilon \mid \mathrm{I}_{k-1}\right)}{p_{\delta}} \mathrm{d} \epsilon \\
= & \frac{1}{p_{\delta}} \tilde{g}\left(P_{k}^{-}\right) \int_{\Omega} f_{\epsilon_{k}}\left(\epsilon \mid \mathrm{I}_{k-1}\right) \mathrm{d} \epsilon \\
= & \tilde{g}\left(P_{k}^{-}\right),
\end{aligned}
$$

where to get the second last equality, we note that from (27) we have $\int_{\Omega} f_{\epsilon_{k}}\left(\epsilon \mid \mathrm{I}_{k-1}\right) \mathrm{d} \epsilon=p_{\delta}$.

Proof to Theorem 3.2: The proof of the time update is simple:

$$
\begin{aligned}
\hat{x}_{k}^{-} & =A \mathbb{E}\left[\mathrm{x}_{k-1} \mid \mathrm{I}_{k-1}\right]=A \hat{x}_{k-1}, \\
P_{k}^{-} & =\mathbb{E}\left[\left(A \mathrm{e}_{k-1}+w_{k-1}\right)\left(A \mathrm{e}_{k-1}+w_{k-1}\right)^{\prime} \mid \mathrm{I}_{k-1}\right] \\
& =A P_{k-1} A^{\prime}+Q=h\left(P_{k-1}\right) .
\end{aligned}
$$

Next, we verify the measurement update for the following two cases.

1) $\gamma_{k}=1$ : According to (26) and (28),

$$
\begin{aligned}
\hat{x}_{k} & =\hat{x}_{k}^{-}+L_{k} z_{k}, \\
P_{k} & =\tilde{g}\left(P_{k}^{-}\right) .
\end{aligned}
$$

2) $\gamma_{k}=0$ : the sensor does not send $y_{k}$ to the remote estimator which computes $\hat{x}_{k}$ as

$$
\begin{aligned}
\hat{x}_{k} & =\mathbb{E}\left[\mathrm{x}_{k} \mid \hat{\mathrm{I}}_{k}\right] \\
& =\frac{1}{p_{\delta}} \int_{\Omega} \mathbb{E}\left[\mathrm{x}_{k} \mid \mathrm{I}_{k-1}, \mathrm{z}_{\mathrm{k}}=F_{k}^{\prime-1} \epsilon\right] f_{\epsilon_{k}}\left(\epsilon \mid \mathrm{I}_{k-1}\right) \mathrm{d} \epsilon \\
& =\frac{1}{p_{\delta}} \int_{\Omega}\left(\hat{x}_{k}^{-}+L_{k} F_{k}^{\prime-1} \epsilon\right) f_{\epsilon_{k}}\left(\epsilon \mid \mathrm{I}_{k-1}\right) \mathrm{d} \epsilon \\
& =\hat{x}_{k}^{-}+\frac{L_{k} F_{k}^{\prime-1}}{p_{\delta}} \int_{\Omega} \epsilon f_{\epsilon_{k}}\left(\epsilon \mid \mathrm{I}_{k-1}\right) \mathrm{d} \epsilon \\
& =\hat{x}_{k}^{-},
\end{aligned}
$$

where the last equality is due to that

$$
\int_{\Omega} \epsilon f_{\epsilon_{k}}\left(\epsilon \mid \mathrm{I}_{k-1}\right) \mathrm{d} \epsilon=0
$$

since being a pdf of Gaussian distribution, $f_{\epsilon_{k}}\left(\epsilon \mid \mathrm{I}_{k-1}\right)$ is even and $\Omega$ defined in (12) is symmetric and centered in the origin. Now from (23), (25), Lemmas 3.3 and A.1, the corresponding error covariance matrix $P_{k}$ can be computed as

$$
\begin{aligned}
P_{k}= & \mathbb{E}\left[\left(\mathrm{x}_{k}-\hat{x}_{k}\right)\left(\mathrm{x}_{k}-\hat{x}_{k}\right)^{\prime} \mid \hat{\mathrm{I}}_{k}\right] \\
= & \mathbb{E}\left[\left(\mathrm{x}_{k}-\hat{x}_{k}^{-}\right)\left(\mathrm{x}_{k}-\hat{x}_{k}^{-}\right)^{\prime} \mid \hat{\mathrm{I}}_{k}\right] \\
= & \mathbb{E}\left[\left\{\left(\mathrm{e}_{k}^{-}-L_{k} \mathrm{z}_{k}\right)+L_{k} \mathrm{z}_{k}\right\}\left\{\left(\mathrm{e}_{k}^{-}-L_{k} \mathrm{z}_{k}\right)+L_{k} \mathrm{z}_{k}\right\}^{\prime} \mid \hat{\mathrm{I}}_{k}\right] \\
= & \mathbb{E}\left[\left(\mathrm{e}_{k}^{-}-L_{k} \mathrm{z}_{k}\right)\left(\mathrm{e}_{k}^{-}-L_{k} \mathrm{z}_{k}\right)^{\prime}+\left(\mathrm{e}_{k}^{-}-L_{k} \mathrm{z}_{k}\right) \mathrm{z}_{k}^{\prime} L_{k}^{\prime}\right. \\
& \left.\quad+L_{k} \mathrm{z}_{k}\left(\mathrm{e}_{k}^{-}-L_{k} \mathrm{z}_{k}\right)^{\prime}+L_{k} \mathrm{z}_{k} \mathrm{z}_{k}^{\prime} L_{k}^{\prime} \mid \hat{\mathrm{I}}_{k}\right] \\
= & \tilde{g}\left(P_{k}^{-}\right)+L_{k} \mathbb{E}\left[\mathrm{z}_{k} \mathrm{z}_{k}^{\prime} \mid \hat{\mathrm{I}}_{k}\right] L_{k}^{\prime} \\
= & \tilde{g}\left(P_{k}^{-}\right)+[1-\beta(\delta)] L_{k}\left(F_{k} F_{k}^{\prime}\right)^{-1} L_{k}^{\prime} \\
= & \tilde{g}\left(P_{k}^{-}\right)+[1-\beta(\delta)] L_{k}\left(C P_{k}^{-} C^{\prime}+R\right) L_{k}^{\prime} \\
= & \tilde{g}_{\beta(\delta)}\left(P_{k}^{-}\right) .
\end{aligned}
$$

From Theorem 3.2, we can write the update for $P_{k}$ in a compact form as

$$
P_{k}= \begin{cases}g\left(P_{k-1}\right), & \text { if } \gamma_{k}=1, \\ g_{\beta(\delta)}\left(P_{k-1}\right), & \text { if } \gamma_{k}=0 .\end{cases}
$$

Remark 3.5. $P_{k}$ is a function of $\left\{\gamma_{t}\right\}_{t=0}^{k}$ and $\beta(\delta)$, both of which depend on $\delta$. By properly tuning $\delta$, we can achieve a desired trade-off between the sensor communication rate $\gamma$ and the estimation quality in terms of $P_{k}$. For example, if we wish to have a small $\gamma$, then picking a large $\delta$ would serve the purpose. The optimal choice of $\delta$ depends on the available communication resources.

Lemma 3.6. Let $\delta \geq 0$. Then

$$
\operatorname{Pr}\left(\left\|\epsilon_{k}\right\|_{\infty} \leq \delta \mid I_{k-1}\right)=[1-2 Q(\delta)]^{m} .
$$

Proof: Note that $\left\|\epsilon_{k}\right\|_{\infty}=\max \left\{\left|\epsilon_{k}^{1}\right|, \ldots,\left|\epsilon_{k}^{m}\right|\right\} \leq \delta$ iff $\left|\epsilon_{k}^{i}\right| \leq \delta, \forall 1 \leq i \leq m$. Therefore,

$$
\begin{aligned}
\operatorname{Pr}\left(\left\|\epsilon_{k}\right\|_{\infty} \leq \delta \mid I_{k-1}\right) & =\prod_{i=1}^{m} \operatorname{Pr}\left(\left|\epsilon_{k}^{i}\right| \leq \delta \mid I_{k-1}\right) \\
& =[1-2 Q(\delta)]^{m}
\end{aligned}
$$

The following result is on the average sensor-to-estimator communication rate $\gamma$. 


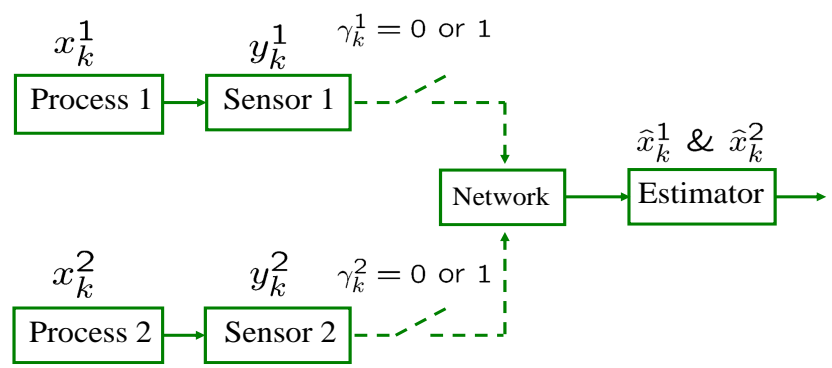

Fig. 2: Scheduling of sensors communication for Process 1 and Process 2

Proposition 3.7. Consider the remote state estimation in Fig. 1 with the event-based sensor scheduler (8). Under the assumption (16), the average sensor-to-estimator communication rate $\gamma$ in (9) is given by

$$
\gamma=1-[1-2 Q(\delta)]^{m}
$$

Proof: Note that $\gamma_{k}$ is a random variable taking value in $\{0,1\}$ with $\operatorname{Pr}\left(\gamma_{k}=1 \mid \mathrm{I}_{k-1}\right)=\operatorname{Pr}\left(\left\|\epsilon_{k}\right\|_{\infty}>\delta \mid \mathrm{I}_{k-1}\right)$. From Lemma 3.6, $\operatorname{Pr}\left(\left\|\epsilon_{k}\right\|_{\infty} \leq \delta \mid I_{k-1}\right)=[1-2 Q(\delta)]^{m}$ whatever value $\mathrm{I}_{k-1}$ takes. Therefore, $\delta$ solely determines the distribution of $\gamma_{k}$ and can be described by

$\operatorname{Pr}\left(\gamma_{k}=0\right)=[1-2 Q(\delta)]^{m}$ and $\operatorname{Pr}\left(\gamma_{k}=1\right)=1-[1-2 Q(\delta)]^{m}$

Equation (30) is then proved from the definition of expectation of $\gamma_{k}$ and the definition of $\gamma$.

\section{Simulation EXAMPLE}

In this example, we consider the scheduling of two sensors measuring Process 1 and Process 2 (see Fig. 2). Let Process 1 be the stable process with $A^{1}=\left[\begin{array}{cc}0.9 & 0.1 \\ 0 & 0.9\end{array}\right], C^{1}=\left[\begin{array}{ll}1 & 0\end{array}\right], Q^{1}=$ $5 I_{2}, R^{1}=2$ and Process 2 be the unstable process with parameters $A^{2}=1.2, C^{2}=1, Q^{2}=10, R^{2}=5$. Assume at each time only one of the sensors is able to communicate its measurement to the remote estimator due to a shortage of communication bandwidth. Since Process 1 is stable, a trivial sensor scheduler $\phi$ that guarantees a bounded estimation error covariance for both processes is that sensor 2 occupies the channel all the time, while sensor 1 is idle and the remote estimator predicts the state of Process 1 at each time. Let the estimation error covariances of Process 1 and Process 2 under the scheduler $\phi$ be $P_{k}^{1}(\phi)$ and $P_{k}^{2}(\phi)$, respectively, which are given by the following two recursions:

$$
P_{k}^{1}=h\left(P_{k-1}^{1}\right) \quad \text { and } \quad P_{k}^{2}=g\left(P_{k}^{2}\right) .
$$

The steady-state values of $\operatorname{Tr}\left\{P_{k}^{1}\right\}$ and $P_{k}^{2}$ under $\phi$ are given by $\operatorname{Tr}\left\{\bar{P}_{1}\right\}=59.00$ and $\bar{P}_{2}=3.77$. By using the event-based scheduler proposed in this paper, we can reduce the estimation error for Process 1 significantly while letting the estimation error for Process 2 grow only slightly. The idea is simple: let sensor 2 follow the event-based scheduler (8); whenever sensor 2 does not send data due to $\left\|\epsilon_{k}^{2}\right\|_{\infty} \leq \delta$, let sensor 1 communicate with the remote estimator. The resulting errors are plotted in Fig. 3 as a function of the parameter $\delta$ being used by Process 2 , which clearly demonstrates the advantage adopting the event-based scheduler. For example, when $\delta=0.4$, the values of $\operatorname{Tr}\left\{\mathbb{E}\left[P_{k}^{1}\right]\right\}^{2}$ and $\mathbb{E}\left[P_{k}^{2}\right]$ are 27.89 and 3.99, respectively, corresponding to a $52.7 \%$ decrease of estimation error

${ }^{2}$ Since $P_{k}$ is a stochastic process due to the randomness of $\gamma_{k}$, we will consider $\operatorname{Tr}\left\{\mathbb{E}\left[P_{k}\right]\right\}$ as a performance measure for the remote estimator under the event-based sensor scheduler (8), which is obtained in this example via Monte Carlo simulations.

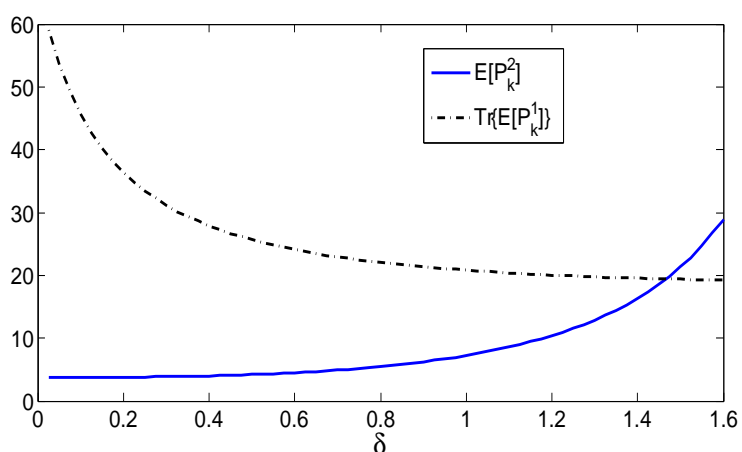

Fig. 3: Estimation quality of Process 1 and Process 2 versus $\delta$

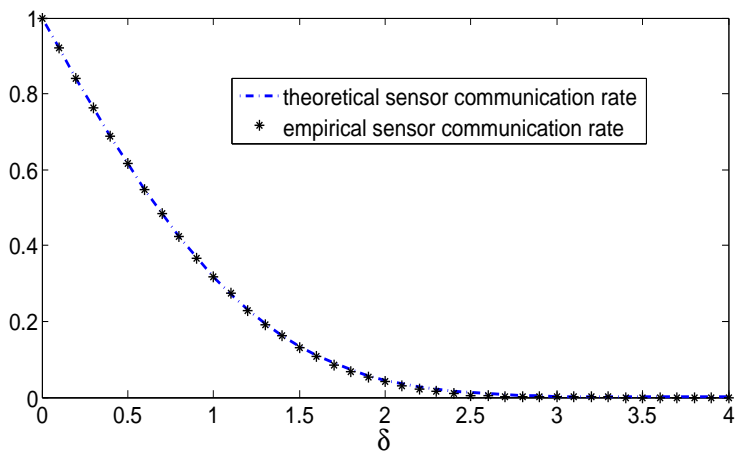

Fig. 4: Sensor communication rate $\gamma$ versus scheduling parameter $\delta$

for Process 1 and a $5.83 \%$ increase of estimation error for Process 2. In Fig. 4, we plot the empirical average sensor communication rate and the theoretical average sensor communication rate (30) for Process 2 under different values of $\delta$. The two curves match almost indistinguishable and demonstrate that the approximated MMSE estimator is very close to the exact MMSE estimator.

\section{CONCLUSION}

We propose an event-based sensor data scheduler for state estimation over a network. The MMSE estimator is derived together with an approximate estimator. It is shown that by tolerating a small amount of increase of the estimation error, a significant reduction of the sensor-to-estimator communication rate can be achieved. In many applications of networked control systems, multiple sensors may be involved. Constructing appropriate event-based schedules at each sensor and estimating the process state based on the received data and the additional information inferred by the events are more difficult than the one we have considered. This will be pursued in our future work.

\section{REFERENCES}

[1] M. Athans, "On the determination of optimal costly measurement strategies for linear stochastic systems," Automatica, vol. 8, no. 4.

[2] V. Gupta, T. Chung, B. Hassibi, and R. M. Murray, "On a stochastic sensor selection algorithm with applications in sensor scheduling and dynamic sensor coverage," Automatica, vol. 42, no. 2, pp. 251-260, 2006.

[3] X. Cao, Stochastic Learning and Optimization: A Sensitivity-Based Approach. Springer-Verlag, 2007.

[4] O. C. Imer and T. Basar, "Optimal estimation with limited measurements," in Proceedings of the 44th IEEE Conference on Decision and Control, European Control, December 2005, pp. 1029-1034.

[5] R. Cogill, S. Lall, and J. P. Hespanha, "A constant factor approximation algorithm for event-based sampling," in Proceedings of the 2007 American Control Conference, New York City, USA, 2007, pp. 305-311.

[6] R. Ambrosino, B. Sinopoli, and K. Poolla, "Optimal sensor scheduling for remote estimation over wireless sensor networks," in Modeling, Estimation and Control of Networked Complex Systems. New York, NY: Springer, 2009, pp. 127-142. 
[7] L. Li, M. Lemmon, and X. Wang, "Event-triggered state estimation in vector linear processes," in Proceedings of the American Control Conference, 2010, pp. 2138-2143.

[8] A. Ribeiro, G. B. Giannakis, and S. I. Roumeliotis, "SOI-KF: Distributed Kalman filtering with low-cost communications using the sign of innovations," IEEE Transactions on Signal Processing, vol. 54, no. 12, pp. 4782-4795, 2006.

[9] E. J. Msechu, S. I. Roumeliotis, A. Ribeiro, and G. B. Giannakis, "Decentralized quantized Kalman filtering with scalable communication cost," IEEE Transactions on Signal Processing, vol. 56, no. 8, pp. 3727$3741,2008$.

[10] B. Anderson and J. Moore, Optimal Filtering. Prentice Hall, 1979.

[11] J. Kotecha and P. Djuric, "Gaussian particle filtering," IEEE Transactions on Signal Processing, vol. 51, no. 10, pp. 2602-2612, 2003.

\section{Appendix}

Lemma A.1: Let $x \in \mathbb{R}$ be a Gaussian r.v. with zero mean and variance $\mathbb{E}\left[\mathrm{x}^{2}\right]=\sigma^{2}$. Denoting $\Delta=\delta \sigma$, then $\mathbb{E}\left[\mathrm{x}^{2}|| \mathrm{x} \mid \leq \Delta\right]=$ $\sigma^{2}(1-\beta(\delta))$.

Proof: The property $f_{\mathrm{x}}(x|| \mathrm{x} \mid \leq \Delta)=\frac{f_{\mathrm{x}}(x)}{\int_{-\Delta}^{\Delta} f_{\mathrm{x}}(t) \mathrm{d} t}$ yields

$$
\begin{aligned}
\mathbb{E}\left[\mathrm{x}^{2}|| \mathrm{x} \mid \leq \Delta\right] & =\frac{1}{\int_{-\Delta}^{\Delta} f_{\mathrm{x}}(t) \mathrm{d} t} \int_{-\Delta}^{\Delta} \frac{t^{2}}{\sqrt{2 \pi}} e^{-\frac{t^{2}}{2 \sigma^{2}}} \mathrm{~d} t \\
& =\frac{\sigma^{2}}{1-2 Q(\delta)} \int_{-\delta}^{\delta} \frac{y^{2}}{\sqrt{2 \pi}} e^{-\frac{y^{2}}{2}} \mathrm{~d} y
\end{aligned}
$$

where $\int_{-\delta}^{\delta} \frac{y^{2}}{\sqrt{2 \pi}} e^{-\frac{y^{2}}{2}} \mathrm{~d} y$ can be calculated as:

$$
\begin{aligned}
\int_{-\delta}^{\delta} \frac{y^{2}}{\sqrt{2 \pi}} e^{-\frac{y^{2}}{2}} \mathrm{~d} y & =-\left.\frac{1}{\sqrt{2 \pi}} y e^{-\frac{y^{2}}{2}}\right|_{-\delta} ^{\delta}+\int_{-\delta}^{\delta} \frac{1}{\sqrt{2 \pi}} e^{-\frac{y^{2}}{2}} \mathrm{~d} y \\
& =1-2 Q(\delta)-\frac{2}{\sqrt{2 \pi}} \delta e^{-\frac{\delta^{2}}{2}} .
\end{aligned}
$$

Then $\mathbb{E}\left[\mathrm{x}^{2}|| \mathrm{x} \mid \leq \Delta\right]=\sigma^{2}(1-\beta(\delta))$.

Lemma A.2 [10], Pages 24-25: Let $\mathrm{x} \in \mathbb{R}^{n}$ and $\mathrm{y} \in \mathbb{R}^{m}$ be jointly Gaussian with mean and variance

$$
m=\left[\begin{array}{c}
\bar{x} \\
\bar{y}
\end{array}\right] \text { and } \Sigma=\left[\begin{array}{cc}
\Sigma_{\mathrm{xx}} & \Sigma_{\mathrm{xy}} \\
\Sigma_{\mathrm{yx}} & \Sigma_{\mathrm{yy}}
\end{array}\right] .
$$

Then $\mathrm{x}$ is conditionally Gaussian given $\mathrm{y}=y$ with $f_{\mathrm{x} \mid \mathrm{y}}(x \mid y)=$ $\mathcal{N}\left(\mu, \Sigma_{\mathrm{xx}}-\Sigma_{\mathrm{xy}} \Sigma_{\mathrm{yy}}^{-1} \Sigma_{\mathrm{yx}}\right)$ where $\mu=\bar{x}+\Sigma_{\mathrm{xy}} \Sigma_{\mathrm{yy}}^{-1}(y-\bar{y})$. 